OPEN ACCESS

Edited by:

Mario Gollwitzer,

Philipps University of Marburg,

Germany

Reviewed by:

Julia Anna Glombiewski,

Philipps University of Marburg,

Germany

Regine Klinger,

Universitätsklinikum

Hamburg-Eppendorf (UKE), Germany

${ }^{*}$ Correspondence:

Luana Colloca

colloca@son.umaryland.edu

Specialty section:

This article was submitted to

Cognition,

a section of the journal

Frontiers in Psychology

Received: 08 November 2016

Accepted: 17 February 2017

Published: 06 March 2017

Citation:

Corsi N and Colloca L (2017) Placebo

and Nocebo Effects: The Advantage

of Measuring Expectations and

Psychological Factors.

Front. Psychol. 8:308.

doi: 10.3389/fpsyg.2017.00308

\section{Placebo and Nocebo Effects: The Advantage of Measuring Expectations and Psychological Factors}

\author{
Nicole Corsi ${ }^{1,2}$ and Luana Colloca ${ }^{1,3,4 *}$ \\ 1 Department of Pain Translational Symptom Science, School of Nursing, University of Maryland, Baltimore, MD, USA, \\ ${ }^{2}$ Department of Neurosciences, Biomedicine and Movement Sciences, University of Verona, Verona, Italy, ${ }^{3}$ Department of \\ Anesthesiology/Psychiatry, School of Medicine, University of Maryland, Baltimore, MD, USA, ${ }^{4}$ Center to Advance Chronic \\ Pain Research, University of Maryland, Baltimore, MD, USA
}

Several studies have explored the predictability of placebo and nocebo individual responses by investigating personality factors and expectations of pain decreases and increases. Psychological factors such as optimism, suggestibility, empathy and neuroticism have been linked to placebo effects, while pessimism, anxiety and catastrophizing have been associated to nocebo effects. We aimed to investigate the interplay between psychological factors, expectations of low and high pain and placebo hypoalgesia and nocebo hyperalgesia. We studied 46 healthy participants using a well-validated conditioning paradigm with contact heat thermal stimulations. Visual cues were presented to alert participants about the level of intensity of an upcoming thermal pain. We delivered high, medium and low levels of pain associated with red, yellow and green cues, respectively, during the conditioning phase. During the testing phase, the level of painful stimulations was surreptitiously set at the medium control level with all the three cues to measure placebo and nocebo effects. We found both robust placebo hypolagesic and nocebo hyperalgesic responses that were highly correlated with expectancy of low and high pain. Simple linear regression analyses showed that placebo responses were negatively correlated with anxiety severity and different aspects of fear of pain (e.g., medical pain, severe pain). Nocebo responses were positively correlated with anxiety sensitivity and physiological suggestibility with a trend toward catastrophizing. Step-wise regression analyses indicated that an aggregate score of motivation (value/utility and pressure/tense subscales) and suggestibility (physiological reactivity and persuadability subscales), accounted for the $51 \%$ of the variance in the placebo responsiveness. When considered together, anxiety severity, NEO openness-extraversion and depression accounted for the $49.1 \%$ of the variance of the nocebo responses. Psychological factors per se did not influence expectations. In fact, mediation analyses including expectations, personality factors and placebo and nocebo responses, revealed that expectations were not influenced by personality factors. These findings highlight the potential advantage of considering batteries of personality factors and measurements of expectation in predicting placebo and nocebo effects related to experimental acute pain.

Keywords: acute pain, anxiety, conditioning, expectation, fear, neuroticism, suggestibility 


\section{INTRODUCTION}

Personality factors can influence placebo and nocebo effects (Colloca and Grillon, 2014; Colagiuri et al., 2015). Factors such as dispositional optimism (Geers et al., 2005, 2007, 2010; Nes and Segerstrom, 2006; Morton et al., 2009), hypnotic suggestibility (De Pascalis et al., 2002), somatic focus (Geers et al., 2006; Johnston et al., 2012), empathy (Colloca and Benedetti, 2009; Hunter et al., 2014; Rütgen et al., 2015a,b), neuroticism (Peciña et al., 2013), altruism (Peciña et al., 2013), social desirability (Gelfland et al., 1965), dopamine-related traits (Schweinhardt et al., 2009), fear of pain (Flaten et al., 2006; Zubieta et al., 2006; Lyby et al., 2010), locus of ego-resilience (Peciña et al., 2013), anxiety (Staats et al., 2001; Ober et al., 2012), pessimism (Geers et al., 2005; Corsi et al., 2016), pain catastrophizing (Vogtle et al., 2013), harm avoidance, and persistence (Corsi et al., 2016) have been linked to placebo and nocebo effects.

In particular, optimism, the active behavioral and mental coping ability of individuals to face adversity, has been liked to proneness to show higher placebo analgesic effects (Geers et al., $2005,2007,2010)$. Attention toward the body, referred as somatic focus, is related to larger placebo analgesic effects and higher positive expectations (Geers et al., 2006). Empathic resonance and concern for others have been linked to placebo analgesia as well (Colloca and Benedetti, 2009; Hunter et al., 2014; Rütgen et al., 2015a,b). Hypnotic susceptibility and responsiveness to verbal suggestions influence placebo analgesia (Huber et al., 2013). Other factors such as Neuroticism-Extraversion-Openness to experience (NEO), NEO Altruism, NEO Straightforwardness, NEO Angry Hostility and Ego-Resiliency, have been coupled with a $25 \%$ variance in behavioral placebo responses to pain and $27 \%$ of the $\mu$-opioid system activation in the nucleus accumbens (Peciña et al., 2013).

Conversely, anxiety (Staats et al., 2001), harm avoidance and persistence (Corsi et al., 2016) and pain catastrophizing (Swider and Babel, 2013; Vogtle et al., 2013) have been associated with nocebo effects. Anxiety and harm avoidance correlate positively with nocebo effects, while optimism and persistence correlate negatively with nocebo effects in the context of the motor system (Corsi et al., 2016). In the present study, our aim was to investigate how distinct positive and negative personality factors estimate the likelihood of placebo and nocebo effects. Moreover, we aimed to establish the relationship among trial-by-trial expectations of pain reduction and increase, and placebo/nocebo effects, and personality. We hypothesized that using aggregated personality factors and expectations would allow us to better estimate placebo and nocebo responses in a laboratory setting using a well-established conditioning model (Colloca et al., 2010).

\section{MATERIALS AND METHODS}

\section{Study Participants}

We recruited 50 participants from Baltimore, MD, USA to enroll a total of 46 healthy participants ( 24 women; 27.41 \pm 1.07 years; see Table 1). Four participants were excluded: two of them did not meet the inclusion criteria and two were unable to discriminate distinct levels of heat thermal stimulation
TABLE 1 | Characteristics of study participants.

\begin{tabular}{ll}
\hline Characteristics of Participants & \\
\hline Sex & 24 females \\
& 22 males \\
Age (years) & $27.41 \pm 1.07$ \\
Body Mass Index (BMI) & $26.00 \pm 0.71$ \\
Systolic blood pressure values $(\mathrm{mmHg})$ & $120.19 \pm 2.00$ \\
Diastolic blood pressure $(\mathrm{mmHg})$ & $75.15 \pm 1.27$ \\
Heart rate (beats per minute) & $66.36 \pm 1.45$ \\
Levels of pain $\left({ }^{\circ} \mathrm{C}\right)$ & Low $41.51 \pm 0.36$ \\
& Medium $44.55 \pm 0.36$ \\
& High $47.52 \pm 0.36$
\end{tabular}

All values are expressed as mean $\pm S E$.

that are used for the acquisition phase of the conditioning paradigm. Upon arrival, participants signed a consent form to study pain modulation. Participants with cardiovascular and neurological diseases, family or personal history of psychiatric conditions, personal history of drug abuse, acute or chronic pain, color blindness, impaired hearing, pregnancy and current use of painkillers and any other medication, were excluded from participating in this study. On the day of the experiment, a toxicology drug test was also performed to exclude any recent use of marijuana, cocaine, opiates such as hydrocodone, oxycodone and hydromorphone, amphetamine, methamphetamine, ecstasy/MDMA and phencyclidine. Participants who reported use of tobacco or nicotine over the last year were also excluded.

This study was carried out in accordance with the recommendations of the UMB Institutional Review Board with written informed consent from all subjects.

All subjects gave written informed consent in accordance with the Declaration of Helsinki. The protocol was approved by the UMB Ethics Committee (Prot \# HP00065783). Due to the use of deception, a debriefing written form was given to each participant at the end of the study participation offering to withdraw the data from the study. None of them opted to do so. Participants were compensated for their participation (\$90).

\section{Pain Assessment}

A well-validated paradigm that has been previously described (Colloca et al., 2010) was used to explore placebo and nocebo responses to a contact heat thermal painful stimulation.

Individual pain sensitivity and tolerance were measured in each participant using the ATS Medoc Pathway system (Medoc Advanced Medical System, Rimat Yishai, Israel). A $3 \times 3 \mathrm{~cm}$ thermode was placed on the dominant forearm as confirmed by the Edinburgh Handedness Inventory. The baseline temperature delivered by the Medoc equipment was $32^{\circ} \mathrm{C}$. Ascending series of stimulations starting from warm sensation to maximum tolerable pain were delivered, while the participant was asked to stop the machine as soon as she felt a warm sensation, low, medium and high pain. Each level was assessed four times and averaged to determine the intensities of stimulations to be used during the acquisition and testing phases of the conditioning paradigm. We defined then the painful stimulations by subtracting 3 and $6^{\circ} \mathrm{C}$ 
starting from the highest reported level of tolerable pain (e.g., 49 and $43^{\circ} \mathrm{C}$ ) so that the levels of stimulation were standardized among participants. The intensities of stimulation were also rated to ensure correspondence to individual experience of low, medium and high pain.

\section{Placebo and Nocebo Manipulation}

Three visual cues (red, yellow, and green) were displayed on a computer placed one meter apart from a chair in a quiet lab. Participants were told that the green, yellow and red lights would anticipate the delivery of a low, medium and high level of pain, respectively.

During the acquisition phase of the classical conditioning paradigm, 18 painful stimulations were delivered at the three levels of pain corresponding to an individual low, medium, and high level of pain in association to six red, six yellow, and six green cues, respectively. Afterwards, during the testing phase, 9 stimulations were paired with the three color cues but the intensity was set at same medium control level in accordance with a previously described paradigm (Colloca et al., 2010). The sequence of the cue presentation was counterbalanced across participants using four distinct sequences. This change in the pain levels allowed us to explore how first-hand experience of low and high pain during the acquisition phase results in placebo and nocebo responses during the testing phase. Participants rated the experienced pain immediately after the painful stimulation using the VAS scale (from $0=$ no pain to $100=$ maximum tolerable pain). Pain reports were collected using Celeritas Fiber Optic Response System (Psychology Software Tools, Inc., Sharpsburg, PA, USA).

Moreover, trial-by-trial expectations were measured. The terms "expectation" and "expectancy" have been often used in an interchangeable way. Herein, we adopted the term "expectation" to refer to verbalized and measurable constructs as compared to "expectancies" defining psychophysical predictions that can be present without full awareness (i.e., implicit expectancies) (Kube et al., 2017).

Participants were asked to rate their expectations of the upcoming stimulation immediately before the delivery of the thermal stimulation using a VAS anchored from $0=$ no pain to $100=$ maximum tolerable pain.

During each trial, the visual cue was presented for $4 \mathrm{~s}$. Immediately after the presentation of the cue, participants were asked to rate their expectation ( $5 \mathrm{~s}$ ) about the upcoming stimulus. The thermal stimulation lasted for $10 \mathrm{~s}$. Then participants were asked to rate their perceived pain (5s) and an inter-trial interval followed with a variable timing (8-10 s). The procedure and the delivery of painful stimulations were controlled by scripts pre-programmed in Eprime (Psychology Software Tools, Inc., Sharpsburg, PA, USA; version 2.0). To prevent habituation, the presentation of visual cues during both phases was counterbalanced using four preprogramed sequences.

\section{Psychological Questionnaires}

Participants completed a comprehensive battery of psychological questionnaires, which were chosen to cover distinct psychological factors that we hypothesized to be linked to placebo and nocebo effects. In particular, for the placebo-related factors, we included optimism, reward, suggestibility, empathy and sensation-seeking and motivation. We used the following questionnaires: (1) LifeOrientation Test-Revisited, Lot-R (Scheier et al., 1994) to assess generalized optimism vs. pessimism; (2) Behavioral Inhibition and Behavioral Activation Scale, BIS/BAS (Carver and White, 1994) to investigate dispositional sensitivity to the behavioral inhibition system (BIS) and the behavioral activation system (BAS); (3) Multidimensional Iowa Suggestibility Scale, MISS (Kotov et al., 2004) to investigate the main components of suggestibility; (4) Interpersonal Reactivity Index, IRI (Davis, 1980) to measure the participant's dispositional empathy in different situations; (5) Sensation Seeking (SS) (Zuckerman, 1994) to measure the necessity to find and experience new situations; (6) Tri-dimensional Personality Questionnaire, TPQ (Cloninger et al., 1991) to assess novelty seeking (NS), harm avoidance (HA), and reward dependence (RD); (7) and the Intrinsic Motivation Inventory (IMI) (Markland and Hardy, 1997) to assess participants' experience during the experimental procedure that was just performed.

For the nocebo-related psychological factors included measurements of various aspects of anxiety (e.g., state, severity, and sensitivity), catastrophizing, neuroticism, fear of pain, depression and feelings of worry. The following inventories were used: (1) State and Trait Anxiety Inventory, STAI (Spielberger, 1983) to investigate anxiety either in a precise moment (STAI-Y1) or as a general tendency (STAI-Y2); (2) Anxiety Sensitivity Index, ASI (Reiss et al., 1986) to assessed beliefs of sensations that could have harmful consequences; (3) Beck Anxiety Inventory, BAI (Beck et al., 1988) to measure experience of anxiety symptoms during the previous 2 weeks; (4) Beck Depression Inventory, BDI (Beck et al., 1961) to include items relating to depression, cognitions, as well as physical symptoms; (5) Mood and Anxiety Symptom Questionnaire, MASQ (Haigh et al., 2011) to assess depressive symptoms and anxiety symptoms; (6) Pain Catastrophizing Scale, PCS (Sullivan et al., 1995) to assess catastrophizing impacts on pain experience; (7) Neuroticism-ExtroversionOpenness Inventory (NEO)-Five Factory Inventory (FFI) (Costa and McCrae, 1985, 1992) to investigate Neuroticism, Extraversion, Openness to Experience, Agreeableness, and Conscientiousness; (8) Fear of Pain Questionnaire, FOP (Osman et al., 2002) to measure fear levels to different types of physical pain; (9) Penn State Worry Questionnaire, PSWQ (Meyer et al., 1990) to measure the trait of worry in different situations.

We also administered the Positive and Negative Affective Schedule, PANAS (Crawford and Henry, 2004), that investigates the relationships between positive and negative affect with personality states and emotions.

\section{Statistical Analysis}

VAS pain and VAS expectations ratings were compared using repeated measure ANOVA. We tested for the main effect of the factor condition (red, yellow, and green) and time (trials) set both as within-subjects factors. F-tests were followed by the Bonferroni post-hoc tests for multiple comparisons. We also 
tested for sex influences on placebo and nocebo effects using sex as a between factor. Partial eta squared $\left(\eta^{2}\right)$ effect sizes are reported for all the comparisons.

VAS pain and expectation scores from the testing phase were further averaged across trials to calculate the difference between yellow-green and yellow-red pain scores to be correlated with placebo and nocebo effects, respectively.

The above psychological questionnaire scores were used in both simple correlation and multivariate analyses. We analyzed psychological questionnaire scores using both Spearman correlation and stepwise multiple regression model analyses in which the questionnaires were modeled to predict placebo and nocebo responses. Mediation analyses were also calculated with expectation as mediator (M), placebo (or nocebo) responses as dependent variable $(\mathrm{Y})$, and personality factors as independent variable $(\mathrm{X})$. For testing indirect effects, a bootstrapping method based on resampling of 1,000 times was used in accordance with Preacher and Hayes methods (Preacher and Hayes, 2004; Hayes and Preacher, 2010). All the analyses were carried out using the SPSS software package (SSPS Inc, Chicago, Illinois, USA, vers.21). To minimize alpha errors, the level of significance was set at $p \leq 0.005$.

\section{RESULTS}

We performed separate analyses for the VAS pain and expectation reports related to the acquisition and testing phases of the conditioning paradigm.

\section{Conditioning: Acquisition Phase}

We analyzed the VAS pain reports during the acquisition phase, and found that participants distinguished the low, medium and high levels of painful stimuli [main effect of condition: $F_{(2,88)}$ $\left.=503.970, p<0.001, \eta^{2}=0.920\right]$. The average pain score for red-associated stimuli was $74.73 \pm 2.36$ using an average intensity of pain equal to $47.52^{\circ} \mathrm{C}$, the average pain score for yellow was $29.55 \pm 1.54$ using an average pain equal to $44.55^{\circ} \mathrm{C}$ and the average pain score for green was $9.37 \pm 0.96$ when an average pain equal to $41.51^{\circ} \mathrm{C}$ out of $50^{\circ} \mathrm{C}$ was delivered. The factor time was significant $\left[F_{(5,220)}=7.359, p<0.001, \eta^{2}=0.143\right]$. The condition $\times$ time interaction was significant $\left[F_{(10,440)}=5.324, p\right.$ $<0.001, \eta^{2}=0.108$ ] (Figure 1A) showing a quadratic trajectory $\left[F_{(1,44)}=10.308, p<0.002, \eta^{2}=0.190\right]$.

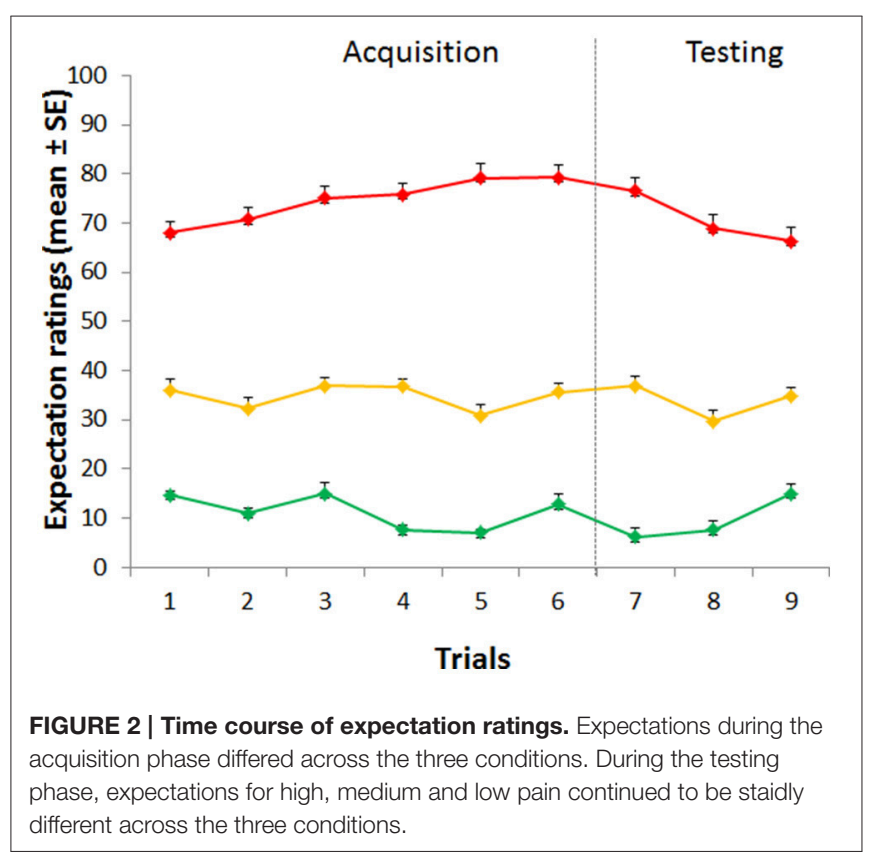

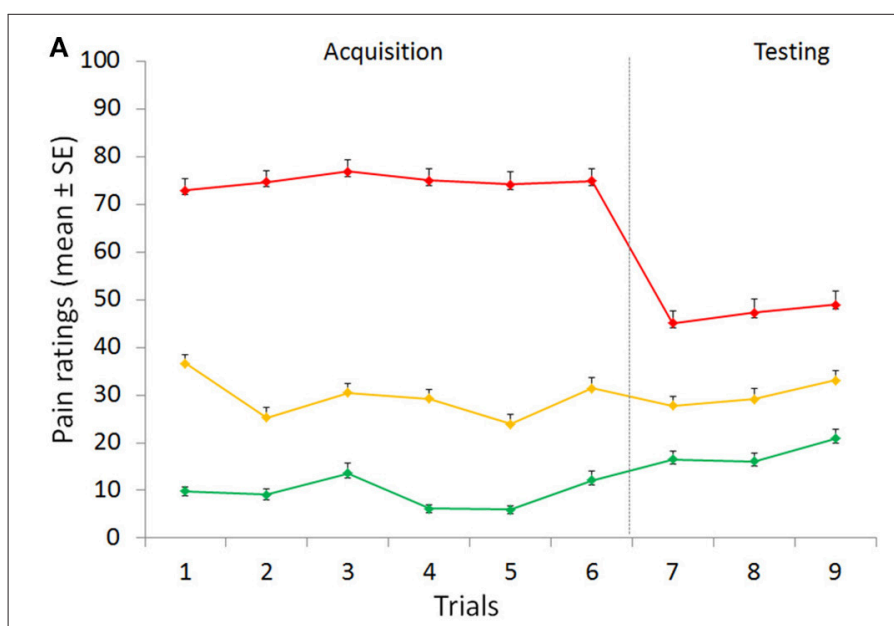
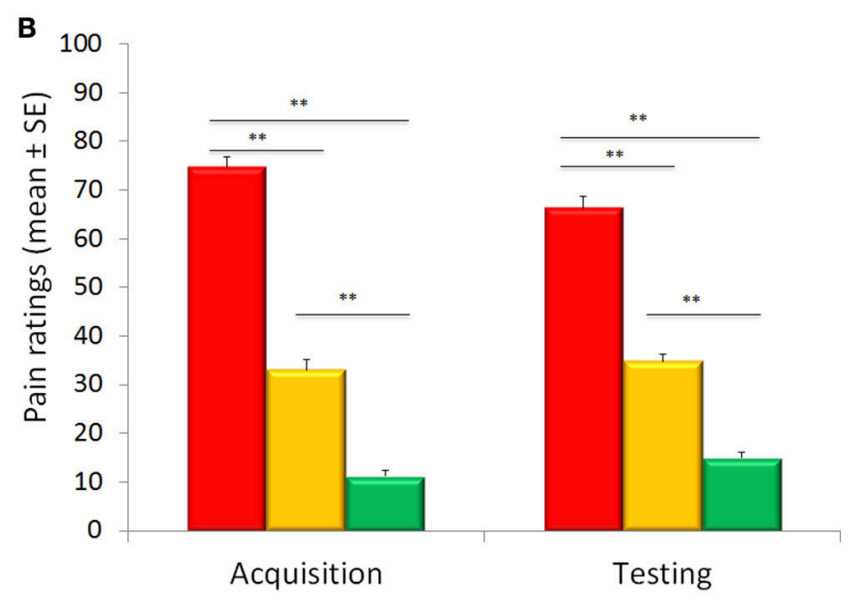

FIGURE 1 | Time course of placebo and nocebo responses (A). Representation trial-by-trial of the average of pain ratings for control (yellow), placebo (green) and nocebo (red) responses during the acquisition (trials 1-6) and the testing (trials 7-9) phases. Participants learned to distinguish the low, medium and high levels of painful stimuli over the acquisition phase. During the testing phase, there was a significant placebo and nocebo effect indicating no extinction over the entire experimental session. Graphical representation of the pain score for the red, green, and yellow associated stimuli (B). The red associated stimuli were perceived as higher than the yellow control stimuli and green were rated as lower than the yellow stimuli during the testing phases when the stimulation was surreptitiously set at a medium level for the three colors indicating both robust placebo and nocebo effects. Data are expressed as mean \pm sem. ${ }^{* *} p<0.001$. 
VAS expectation scores $(75.63 \pm 2.09,34.74 \pm 1.61$, and $11.30 \pm 0.98$, respectively) during the acquisition phase differed across the three conditions $\left[F_{(2,88)}=515.152\right.$, $\left.p<0.001 ; \eta^{2}=0.921\right]$, with significant time $\left[F_{(5,220)}\right.$ $\left.=3.392, p=0.006 ; \eta^{2}=0.072\right]$ and condition $\times$ time interaction $\left[F_{(10,440)}=7.542, p<0.001 ; \eta^{2}=0.146\right]$ effects (Figure 2).

\section{Conditioning: Testing Phase}

During the testing phase, when the level of pain was set at the same control (yellow) intensity for the three cues, VAS pain reports revealed a significant effect of condition $\left[F_{(2,88)}\right.$

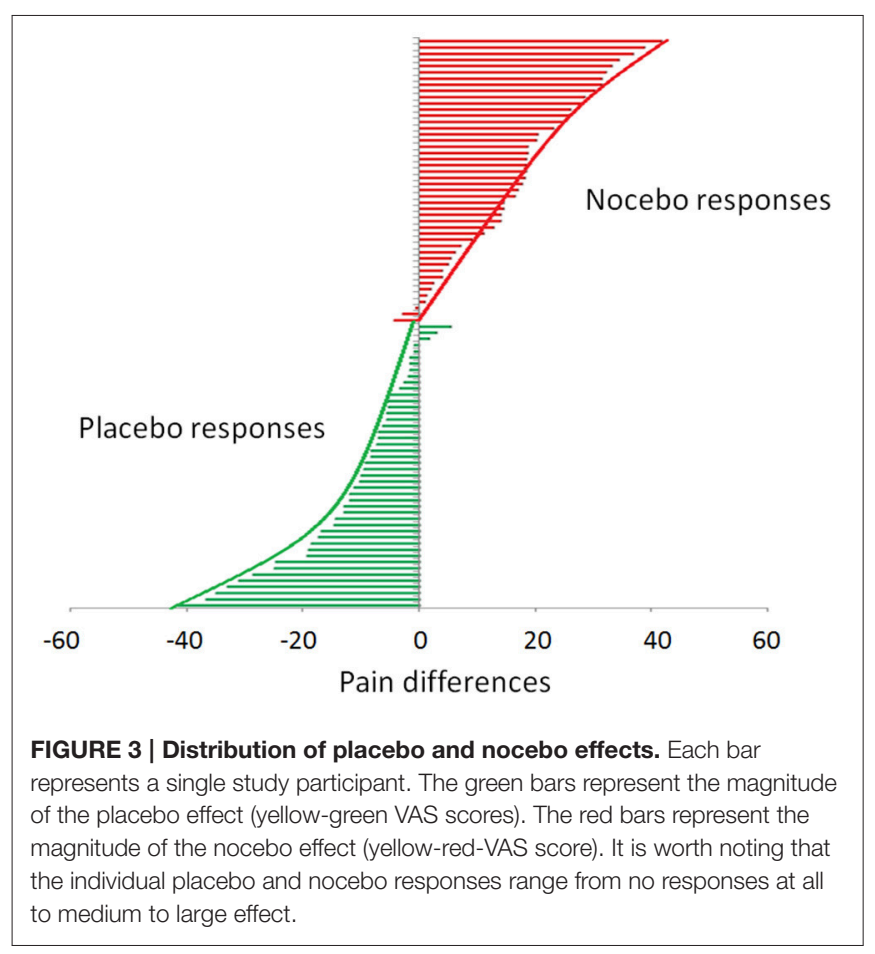

$\left.=96.04, p<0.001 ; \eta^{2}=0.686\right]$, time $\left[F_{(2,88)}=7.553\right.$, $\left.p=0.001 ; \eta^{2}=0.147\right]$ with a non-significant condition $\times$ time interaction $\left[F_{(4,176)}=0.378, p=0.824 ; \eta^{2}=0.009\right]$ indicating no extinction over the entire experimental session (Figure 1A). Post-hoc Bonferroni tests indicated that the red stimuli (average VAS: $46.98 \pm 2.46$ ) were perceived as higher than the yellow control stimuli (average VAS: $29.96 \pm 1.78$ ) $(p<0.001)$ and green (average VAS: $17.86 \pm 1.70)$ were rated as lower than the yellow stimuli $(p<0.001)$ indicating both robust placebo and nocebo effects (Figure 1B). The distribution and magnitude of placebo and nocebo responses ranged from no effects to large changes in pain modulation (Figure 3).

Placebo effects were significantly correlated with the hypoalgesic effect experienced during the acquisition phase (Placebo: $r=0.388, p=0.008$ ) but nocebo hyperalgesic responses appeared to be independent of the experienced high pain $(r=0.080, p=0.598)$. Moreover, being prone to experience a placebo response did not imply being also prone to experience a nocebo response, as indicated by the absence of significant correlation between individual placebo and the nocebo responses $(r=-0.113$, $p=0.454)$.

During the testing phase, expectations for high, medium and low pain $[70.61 \pm 2.45,33.87 \pm 1.81$, and $9.54 \pm 0.93]$ were different across the three conditions $\left[F_{(2,88)}=441.355, p<\right.$ $\left.0.001 ; \eta^{2}=0.909\right]$ with a main effect of time $\left[F_{(2,88)}=8.092, p=\right.$ $\left.0.001 ; \eta^{2}=0.155\right]$, and a significant interaction condition $\times$ time $\left[F_{(4,176)}=13.156, p<0.001 ; \eta^{2}=0.230\right]$ (Figure 3), showing a linear trajectory $\left[F_{(1,44)}=33.850, p<0.001, \eta^{2}=0.435\right]$. Importantly, we found that positive expectations correlated with placebo responses $(r=0.412, \mathrm{p}=0.002$, Figure $4 \mathrm{~A})$ and similarly negative expectations correlated with nocebo effects $(r=0.351$, $p=0.008$, Figure 4B).

In this cohort of participants, sex effects for placebo, nocebo and expectancies were not observed [placebo: $F_{(1,44)}=0.010$, $p=0.922$; nocebo: $F_{(1,44)}=0.990, p=0.325$; positive

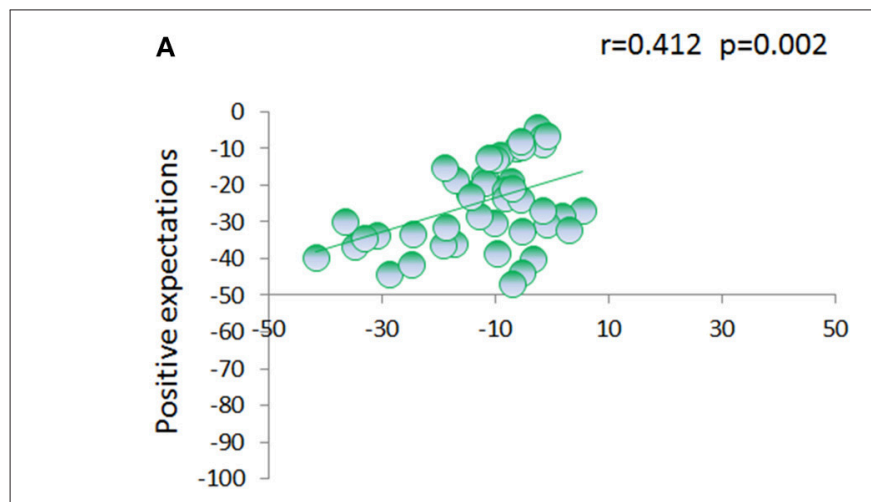

Placebo responses

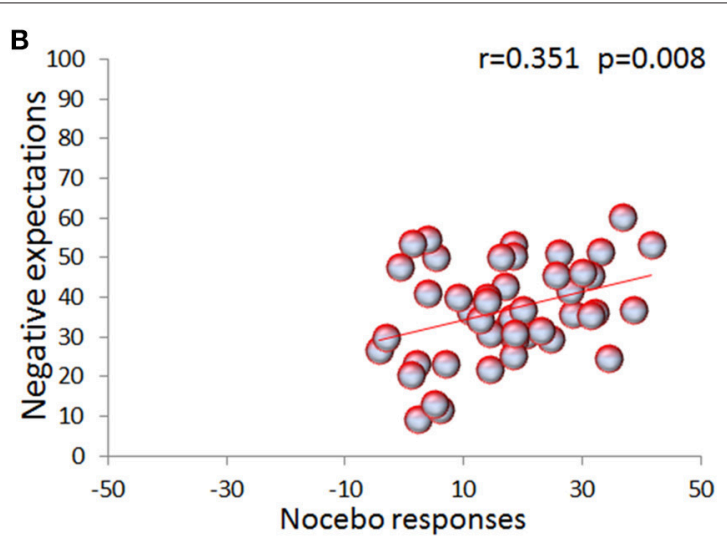

FIGURE 4 | Relation between expectations and placebo/nocebo effects. VAS expectation scores were collected on a trial-by-trial basis during the testing phase. Expectation of low pain positively correlates with placebo effects (A). Similarly, expectation of upcoming high painful stimulation positively correlates with nocebo effects (B). 
TABLE 2 | Correlations between placebo, nocebo and personality factors.

\begin{tabular}{|c|c|c|c|c|}
\hline \multirow[t]{2}{*}{ Personality factors } & \multicolumn{2}{|c|}{ PLACEBO EFFECT } & \multicolumn{2}{|c|}{ NOCEBO EFFECT } \\
\hline & $\boldsymbol{R}$ & $p$ & $r$ & $p$ \\
\hline STAI-Y1 pre & -0.175 & 0.246 & 0.217 & 0.147 \\
\hline STAI-Y2 post & -0.004 & 0.978 & 0.177 & 0.241 \\
\hline STAY2 & -0.123 & 0.415 & 0.119 & 0.432 \\
\hline ASI & -0.147 & 0.330 & 0.460 & 0.001 \\
\hline $\mathrm{BAl}$ & -0.485 & 0.001 & -0.028 & 0.855 \\
\hline BDI & -0.039 & 0.796 & 0.244 & 0.102 \\
\hline PANAS total & 0.096 & 0.527 & 0.175 & 0.245 \\
\hline PANAS positive & -0.218 & 0.145 & -0.199 & 0.429 \\
\hline PANAS negative & -0.366 & 0.012 & 0.248 & 0.097 \\
\hline NEO neuroticism & -0.166 & 0.270 & 0.021 & 0.892 \\
\hline NEO extraversion & -0.349 & 0.018 & 0.186 & 0.217 \\
\hline NEO openness & -0.228 & 0.128 & -0.264 & 0.076 \\
\hline NEO agreeableness & -0.098 & 0.518 & 0.091 & 0.548 \\
\hline NEO conscientiousness & -0.001 & 0.993 & 0.054 & 0.720 \\
\hline MASQ General depressive scale & -0.75 & 0.622 & -0.051 & 0.735 \\
\hline MASQ anxious arousal & 0.021 & 0.889 & -0.098 & 0.519 \\
\hline MASQ general distress & -0.146 & 0.332 & 0.155 & 0.305 \\
\hline MASQ anhedonia & 0.015 & 0.620 & 0.151 & 0.316 \\
\hline TPQ novelty seeking & 0.015 & 0.922 & 0.045 & 0.769 \\
\hline TPQ harm avoidance & -0.164 & 0.277 & 0.099 & 0.511 \\
\hline TPQ reward dependence & -0.191 & 0.204 & 0.179 & 0.234 \\
\hline BAS drive & 0.165 & 0.273 & -0.016 & 0.918 \\
\hline BAS fun & 0.000 & 0.999 & -0.139 & 0.356 \\
\hline BAS reward & 0.004 & 0.977 & -0.32 & 0.382 \\
\hline BIS & -0.065 & 0.670 & -0.091 & 0.549 \\
\hline BIS/BAS total & 0.013 & 0.934 & -0.144 & 0.341 \\
\hline LotR & -0.072 & 0.635 & 0.012 & 0.935 \\
\hline IMI interest/enjoyment & -0.075 & 0.620 & -0.081 & 0.595 \\
\hline IMI perceived competence & -0.280 & 0.060 & -0.022 & 0.883 \\
\hline IMl effort/importance & -0.142 & 0.346 & -0.220 & 0.143 \\
\hline IMI pressure/tense & -0.017 & 0.910 & 0.295 & 0.047 \\
\hline IMI choice & -0.094 & 0.540 & -0.197 & 0.194 \\
\hline IMI value/utility & -0.343 & 0.020 & -0.216 & 0.149 \\
\hline IMI total & -0.339 & 0.021 & -0.144 & 0.341 \\
\hline IRI fantasy & -0.290 & 0.050 & -0.016 & 0.915 \\
\hline IRI empathic concern & -0.231 & 0.123 & 0.157 & 0.297 \\
\hline IRI perspective-taking & -0.189 & 0.208 & 0.093 & 0.538 \\
\hline $\mid \mathrm{RI}$ personal distress & 0.190 & 0.207 & 0.195 & 0.193 \\
\hline MISS suggestibility & -0.165 & 0.274 & 0.254 & 0.089 \\
\hline MISS persuadability & -0.060 & 0.693 & -0.016 & 0.918 \\
\hline MISS physiological suggestibility & -0.264 & 0.076 & 0.438 & 0.002 \\
\hline MISS physiological reactivity & -0.354 & 0.016 & 0.159 & 0.292 \\
\hline MISS peer conformity & -0.270 & 0.069 & 0.293 & 0.048 \\
\hline MISS mental control & -0.220 & 0.141 & 0.005 & 0.975 \\
\hline MISS unpersuadability & -0.121 & 0.421 & 0.295 & 0.047 \\
\hline MISS short suggestibility & -0.284 & 0.056 & 0.175 & 0.245 \\
\hline MISS total & -0.331 & 0.025 & 0.301 & 0.042 \\
\hline FOP severe & -0.490 & 0.001 & -0.073 & 0.629 \\
\hline FOP medical & -0.416 & 0.004 & -0.013 & 0.929 \\
\hline
\end{tabular}

(Continued)
TABLE 2 | Continued

\begin{tabular}{lrrrrr}
\hline Personality traits & \multicolumn{2}{c}{ PLACEBO EFFECT } & & \multicolumn{2}{c}{ NOCEBO EFFECT } \\
\cline { 2 - 3 } \cline { 5 - 6 } & \multicolumn{1}{c}{$\boldsymbol{R}$} & $\boldsymbol{p}$ & & $\boldsymbol{r}$ & $\boldsymbol{p}$ \\
\hline FOP total & $-\mathbf{0 . 4 3 5}$ & $\mathbf{0 . 0 0 3}$ & & -0.037 & 0.806 \\
PCS rumination & -0.104 & 0.490 & & 0.352 & 0.016 \\
PCS magnification & 0.032 & 0.831 & & 0.054 & 0.721 \\
PCS helplessness & 0.021 & 0.887 & & 0.366 & 0.012 \\
PCS total & -0.022 & 0.883 & & 0.343 & 0.020 \\
PSWQ & -0.216 & 0.149 & & 0.283 & 0.057 \\
SS boredom susceptibility & -0.066 & 0.661 & & 0.098 & 0.518 \\
SS disinhibition & -0.031 & 0.839 & & -0.027 & 0.861 \\
SS experience seeking & 0.078 & 0.605 & -0.093 & 0.537 \\
SS adventure seeking & -0.036 & 0.812 & -0.025 & 0.869 \\
SS total & 0.014 & 0.924 & & 0.005 & 0.976
\end{tabular}

STAI 1-2, State and Trait Anxiety Inventory; ASI, Anxiety Sensitivity Index; BAl, Beck Anxiety Inventory; BDI, Beck Depression Inventory; PANAS, Positive and Negative Affective Schedule; NEO, Neuroticism-Extraversion-Openness Inventory; MASQ, Mood and Anxiety Symptom Questionnaire; TPQ, Tridimensional Personality Questionnaire; BISBAS, Behavioral Inhibition and Behavioral Activation Scale; LotR, Life-Orientation TestRevisited; IMI, Intrinsic Motivation Inventory; IRI, Interpersonal Reactivity Index; MISS, Multidimensional Iowa Suggestibility Scale; FOP, Fear of Pain; PCS, Pain Catastrophizing Scale; PSWQ, Penn State Worry Questionnaire; SS, Sensation Seeking. Significant results are indicated in bold.

expectancies: $F_{(1,44)}=1.860, p=0.180$; negative expectancies: $\left.F_{(1,44)}=0.025, p=0.875\right]$.

\section{Personality Predictors}

We then explored the effects of personality factors on placebo and nocebo effects. First, we ran a series of correlations analyses and found that placebo responses were negatively correlated with severity of anxiety (BAI: $r=-0.485, p=0.001$ ), and fear of pain (FOP, severe: $r=-0.490, p=0.001$; medical fear, $r=-0.416, p=$ 0.004 ; total fear $r=-0.435, p=0.003$ ). By the contrary, nocebo responses were positively correlated with anxiety sensitivity (ASI, $r=0.460, p=0.001$ ), physiological suggestibility (MISS: $r=$ $0.438, p=0.002$ ) with a trend for catastrophizing tendency (PCS rumination: $r=0.352, p=0.016$; PCS helplessness: $r=0.366$, $p=0.012$; PCS total: $r=0.343, p=0.020)$ (Table 2).

Moreover, we considered the hypothesized psychological factors taken together in order to identify their relationship with the dependent variables (e.g., placebo and nocebo VAS) using stepwise multiple regression models. The significant values are reported in Tables 3, 4. Motivation (value/utility and pressure/tense subscales) and suggestibility (physiological reactivity and persuadability subscales) accounted for $51 \%$ of variance in placebo responses (Table 3). Conversely, ASI, NEOopenness-extraversion and depression taken together accounted for $49.1 \%$ of variance in nocebo responses (Table 4).

Finally, we calculated mediation analyses for exploring the relationship among personality factors, positive/negative expectations and placebo/nocebo responses. Interestingly, we found that expectations were significantly linked to placebo and nocebo effects (see Table 5). However, personality factors per se did not influence expectancies, and the indirect effect among the three variables was not significant. Due to the exploratory 
TABLE 3 | Stepwise multiple regression models for the prediction of placebo effects.

\begin{tabular}{|c|c|c|c|c|c|}
\hline Dependent variable & Predictor Variables & $R^{2}$ & $\beta$ & $t$ & $p$ \\
\hline \multirow[t]{2}{*}{ Placebo hypoalgesia } & Model 1 & 21.6 & & & \\
\hline & MISS physiol & & 0.464 & 3.438 & 0.001 \\
\hline \multirow[t]{5}{*}{ Placebo hypoalgesia } & Model 1 & 21.6 & & & \\
\hline & MISS physiol & & 0.464 & 3.438 & 0.001 \\
\hline & Model 2 & 35.4 & & & \\
\hline & MISS physiol & & 0.577 & 4.452 & $<0.001$ \\
\hline & IMI value & & -0.389 & -2.999 & 0.005 \\
\hline \multirow[t]{9}{*}{ Placebo hypoalgesia } & Model 1 & 21.6 & & & \\
\hline & MISS physiol & & 0.464 & 3.438 & 0.001 \\
\hline & Model 2 & 35.4 & & & \\
\hline & MISS physiol & & 0.577 & 4.452 & $<0.001$ \\
\hline & IMI value & & -0.389 & -2.999 & 0.005 \\
\hline & Model 3 & 42.7 & & & \\
\hline & MISS physiol & & 0.579 & 4.687 & $<0.001$ \\
\hline & IMl value & & -0.371 & -2.993 & 0.005 \\
\hline & MISS persuadability & & 0.270 & 2.280 & 0.028 \\
\hline \multirow[t]{14}{*}{ Placebo hypoalgesia } & Model 1 & 21.6 & & & \\
\hline & MISS physiol & & 0.464 & 3.438 & 0.001 \\
\hline & Model 2 & 35.4 & & & \\
\hline & MISS physiol & & 0.577 & 4.452 & $<0.001$ \\
\hline & IMl value & & -0.389 & -2.999 & 0.005 \\
\hline & Model 3 & 42.7 & & & \\
\hline & MISS physiol & & 0.579 & 4.687 & $<0.001$ \\
\hline & IMI value & & -0.371 & -2.993 & 0.005 \\
\hline & MISS persuadability & & 0.270 & 2.280 & 0.028 \\
\hline & Model 4 & 51.0 & & & \\
\hline & MISS physiol & & 0.463 & 3.745 & 0.001 \\
\hline & IMl value & & -0.335 & -2.871 & 0.007 \\
\hline & MISS persuadability & & 0.344 & 3.006 & 0.005 \\
\hline & IMl pressure & & 0.319 & 2.617 & 0.012 \\
\hline
\end{tabular}

MISS, Multidimensional lowa Suggestibility Scale (Physiological Reactivity and Persuadability subscales); IMI, Intrinsic Motivation Inventory Nalue/Utility and Pressure/Tense subscales). Only significant values are shown. Excluded variables (not significant): Lot-R, Life-Orientation Test-Revisited; BIS/BAS, Behavioral Inhibition and Behavioral Activation Scale; IRI, Interpersonal Reactivity Index; SS, Sensation Seeking; $T P Q$, Tri-dimensional Personality Questionnaire.

nature of this part of the study, we used a relative broad battery. Therefore, correlations among personality questionnaires are shown in Table 6.

\section{DISCUSSION}

In this study, we investigated the influence of expectations and hypothesized psychological factors on placebo and nocebo effects elicited by a well-established model of conditioning and heat thermal painful stimulation. Placebo hypoalgesic responses were negatively correlated with severity of anxiety and fear of pain (e.g., medical fear, severe, and total fear). On the contrary, nocebo hyperalgesic responses were positively correlated with anxiety
TABLE 4 | Stepwise multiple regression models for the prediction of nocebo effects.

\begin{tabular}{|c|c|c|c|c|c|}
\hline Dependent variable & Predictor Variables & $R^{2}$ & $\beta$ & $t$ & $p$ \\
\hline \multirow[t]{2}{*}{ Nocebo hyperalgesia } & Model 1 & 20.3 & & & \\
\hline & ASI & & 0.451 & 3.349 & 0.002 \\
\hline \multirow[t]{5}{*}{ Nocebo hyperalgesia } & Model 1 & 20.3 & & & \\
\hline & ASI & & 0.451 & 3.349 & 0.002 \\
\hline & Model 2 & 33.3 & & & \\
\hline & ASI & & 0.498 & 3.966 & $<0.001$ \\
\hline & NEO_O & & -0.364 & -2.897 & 0.006 \\
\hline \multirow[t]{9}{*}{ Nocebo hyperalgesia } & Model 1 & 20.3 & & & 0.002 \\
\hline & ASI & & 0.451 & 3.349 & \\
\hline & Model 2 & 33.3 & & & \\
\hline & ASI & & 0.498 & 3.966 & $<0.001$ \\
\hline & NEO openess & & -0.364 & -2.897 & 0.006 \\
\hline & Model 3 & 42.9 & & & \\
\hline & ASI & & 0.493 & 4.197 & $<0.001$ \\
\hline & NEO openess & & -0.472 & -3.796 & $<0.001$ \\
\hline & NEO extraversion & & 0.329 & 2.660 & 0.011 \\
\hline \multirow[t]{14}{*}{ Nocebo hyperalgesia } & Model 1 & 20.3 & & & \\
\hline & ASI & & 0.451 & 3.349 & 0.002 \\
\hline & Model 2 & 33.3 & & & \\
\hline & ASI & & 0.498 & 3.966 & $<0.001$ \\
\hline & NEO openess & & -0.364 & -2.897 & 0.006 \\
\hline & Model 3 & 42.9 & & & \\
\hline & ASI & & 0.493 & 4.197 & $<0.001$ \\
\hline & NEO openess & & -0.472 & -3.796 & $<0.001$ \\
\hline & NEO extraversion & & 0.329 & 2.660 & 0.011 \\
\hline & Model 4 & 49.1 & & & \\
\hline & ASI & & 0.448 & 3.919 & $<0.001$ \\
\hline & NEO openess & & -0.413 & -3.388 & 0.002 \\
\hline & NEO extraversion & & 0.387 & 3.197 & 0.003 \\
\hline & BDI & & 0.267 & 2.218 & 0.032 \\
\hline
\end{tabular}

ASI, Anxiety Sensitivity Index; BDI, Beck Depression Inventory; NEO, NeuroticismExtraversion-Openness Inventory. Only significant values are shown. Excluded variables (not significant for the model): STAI, State and Trait Anxiety Inventory; BAl, Beck Anxiety Inventory; FOP. Fear of Pain; MASQ, Mood and Anxiety Symptom Questionnaire; PCS, Pain Catastrophizing Scale; PSWQ, Penn State Worry Questionnaire; PANAS, Positive and Negative Affective Schedule.

sensitivity, suggestibility and catastrophizing (trend only). Moreover, a stepwise regression modeling showed that aggregate scores of Motivation (value/utility and pressure/tense subscales) and suggestibility (physiological reactivity and persuadability subscales) accounted for the $51 \%$ of the variance in the placebo responses. By contrast, the aggregation of anxiety, openness, extraversion and depression accounted for the $49.1 \%$ of the variance in the nocebo responses. Importantly, expectations were highly correlated with placebo and nocebo effects and psychological factors did not influence level of expectations towards reduction or increase of pain.

Consistently with previous studies (Colloca and Benedetti, 2006, 2009; Colloca et al., 2008, 2010; Lui et al., 2010), we found 
TABLE 5 | Mediation analysis results.

\begin{tabular}{lcccc}
\hline Model & a path & b path & c' path & $\begin{array}{c}\text { Indirect } \\
\text { effect }\end{array}$ \\
\hline BAI $(X)$ & $p=0.094$ & $p=0.018$ & $p=0.099$ & $p=0.184$ \\
$\begin{array}{l}\text { Positive expectations }(M) \\
\text { Placebo hypoalgesia }(Y)\end{array}$ & & & & \\
\hline FOP severe $(X)$ & $p=0.656$ & $p=0.005$ & $p=0.005$ & $p=0.673$ \\
$\begin{array}{l}\text { Positive expectations }(M) \\
\text { Placebo hypoalgesia }(Y)\end{array}$ & & & & \\
\hline
\end{tabular}

\begin{tabular}{lllll}
\hline FOP medical $(X)$ & $p=0.656$ & $p=0.011$ & $p=0.012$ & $p=0.309$ \\
Positive expectations $(M)$ & & \\
Placebo hypoalgesia $(M)$ &
\end{tabular}

\begin{tabular}{|c|c|c|c|c|}
\hline FOP total $(X)$ & $p=0.217$ & $p=0.007$ & $p=0.012$ & $p=0.286$ \\
\hline Positive expectations (M) & & & & \\
\hline Placebo hypoalgesia ( $)$ & & & & \\
\hline
\end{tabular}

\begin{tabular}{|c|c|c|c|c|}
\hline MISS physiol. (X) & $p=0.181$ & $p=0.010$ & $p=0.078$ & $p=0.321$ \\
\hline Negative expectations (M) & & & & \\
\hline Nocebo hyperalgesia $(Y)$ & & & & \\
\hline
\end{tabular}

\begin{tabular}{lllll}
\hline ASI $(X)$ & $p=0.871$ & $p<0.001$ & $p=0.014$ & $p=0.879$ \\
Negative expectations $(M)$ & & & \\
Nocebo hyperalgesia $(Y)$ &
\end{tabular}

\begin{tabular}{lllll}
\hline PCS rumination $(X)$ & $p=0.493$ & $p=0.023$ & $p=0.006$ & $p=0.539$ \\
Negative expectations $(M)$ & & & \\
Nocebo hyperalgesia $(M)$ &
\end{tabular}

\begin{tabular}{|c|c|c|c|c|}
\hline PCS help. (X) & $p=0.322$ & $p=0.031$ & $p=0.022$ & $p=0.398$ \\
\hline \multicolumn{5}{|l|}{ Negative expectations (M) } \\
\hline Nocebo hyperalgesia $(Y)$ & & & & \\
\hline
\end{tabular}

\begin{tabular}{lllll}
\hline PCS total $(X)$ & $p=0.350$ & $p=0.027$ & $p=0.014$ & $p=0.419$ \\
Negative expectations $(M)$ & & \\
Nocebo hyperalgesia $(Y)$ &
\end{tabular}

BAI, Beck Anxiety Inventory; FOP, Fear Of Pain; MISS, Multidimensional lowa Suggestibility Scale; ASI, Anxiety Sensitivity Index; PCS, Pain Catastrophizing Scale.

that visual cues associated with prior experiences of low and high pain elicit strong placebo and nocebo effects with a distribution raging from no responses to low modulation of pain, to medium and high reductions and increases (Figure 3). Studies on placebo hypoalgesia and nocebo hyperalgesia have shown a substantial inter-individual variability and distinct personality factors have been associated with placebo and nocebo effects (Colloca and Grillon, 2014; Colagiuri et al., 2015). There is evidence that some personality factors such as anxiety (Staats et al., 2001; Ober et al., 2012), fear of pain (Lyby et al., 2010) and neuroticism (Peciña et al., 2013), are associated with reduced placebo analgesia. We confirmed and expanded some of these findings. In our study, severity of anxiety as well as fear of pain (e.g., medical, sever, and total fear) were linked to reduced placebo responsiveness to pain. Severity of anxiety including symptoms of depression, feelings of hopelessness and irritability, guiltiness or feelings of being punished, as well as physical symptoms such as fatigue, correlated negatively with placebo responses with higher severity of anxiety linked to lower reduction of pain induced by positive expectations. High levels of fear of pain referring to the dispositional tendency to have negative emotions toward pain and pain anticipation have been also associated with placebo- and nocebo-induced pain modulation (Lyby et al., 2010; Aslaksen and Lyby, 2015). We found that fear of medical pain in particular correlates with low placebo hypoalgesic responses and this is consistent with the parallel enhancement of nocebo induced by fear of pain and other medical procedures (Aslaksen and Lyby, 2015).

When we looked at the nocebo effect-the negative counterpart of the placebo phenomenon (Petrovic, 2008)-we found a positive correlation with anxiety sensitivity, physiological suggestibility and catastrophizing. Anxiety sensitivity refers to behaviors or sensations associated with the experience of anxiety that elicit misinterpretations of bodily sensations such as the experience of a no harmful stimulus causing intense pain (Mehta et al., 2016). Suggestibility is a trait-like characteristic creating distinct behaviors that facilitate responsiveness to plausible information as well as inclinations to accept and act on others' suggestions in regards to the body (e.g., physical suggestibility), and has been linked to placebo effects (Lund et al., 2015) and nocebo effects (Corsi et al., 2016). Catastrophizing, a maladaptive cognitive process that is potentially heritable and has been reported to predict severity of clinical pain (Flor and Turk, 1988; Severeijns et al., 2001; Goubert et al., 2004; Kudel et al., 2005; Trost et al., 2015), has been recently explored and shown to be relevant for nocebo effects (Vogtle et al., 2013).

Personality is a continuum of factors and thus highlights the importance of considering distinct factors together. Therefore, based on the literature we took into consideration two sets of psychological factors related to placebo and nocebo responsiveness and used a multilevel modeling approach in which hierarchies and residual components at each level within a hierarchy are computed. Such an approach indicated that an aggregate score for motivation (value/utility and pressure/thanks subscales) and suggestibility (physiological reactivity and persuadability subscales) accounted for the 51\% of the variance in the placebo hypolagesic responses whilst anxiety severity, NEO-openness-extraversion and depression considered together accounted for the $49.1 \%$ of the variance of nocebo responses suggesting that it helps evaluate the psychological factors comprehensively. Another important result from this study was that positive expectations were significantly correlated with placebo responses and negative expectations were significantly correlated with nocebo responses. Although one may argue that asking on a trial-by-trial about expectancy of the upcoming pain may have generated a sort of self-prophecy (e.g., You get what you expect, you get what you ask for), it remains an interesting finding that could be important to keep in mind every time we measure pain in real-world settings. Therefore, an obvious question was whether personality factors impact the formation of expectations of pain reduction and increase. In this study, mediation analyses indicated that personality factors (e.g., being worried, being fearful) had no direct effect on the 


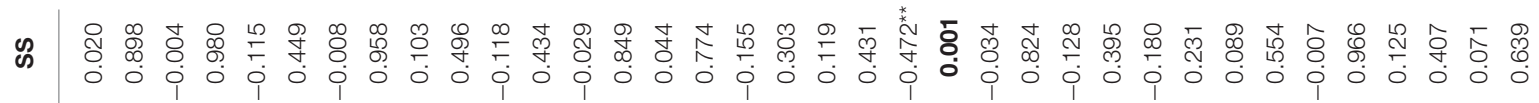

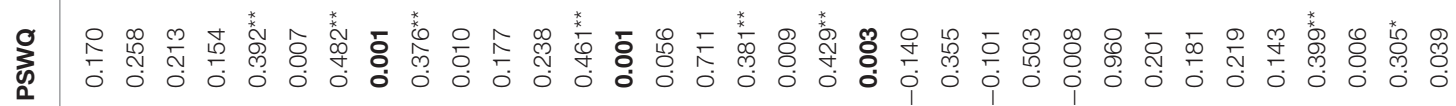

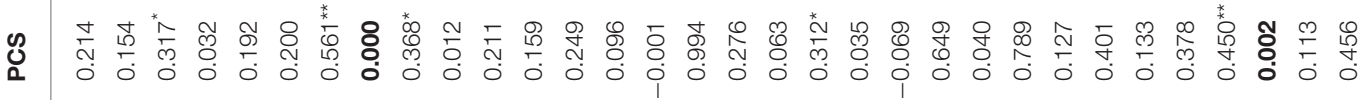

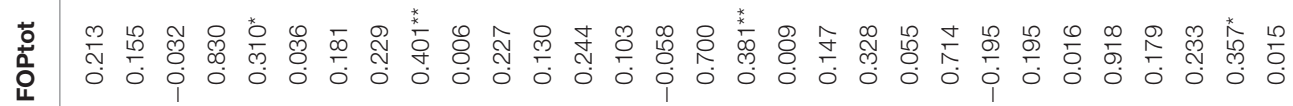

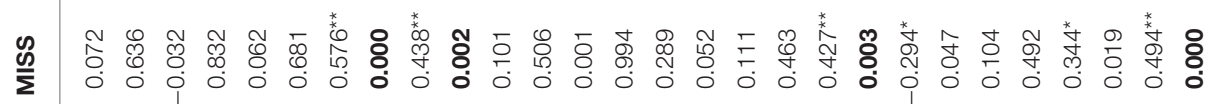

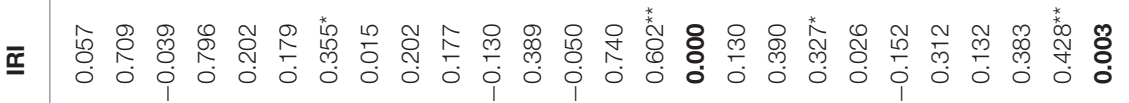

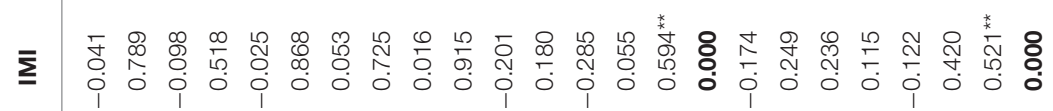

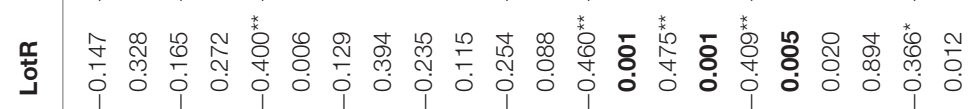

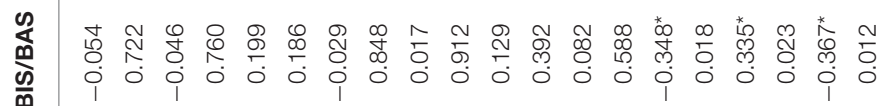

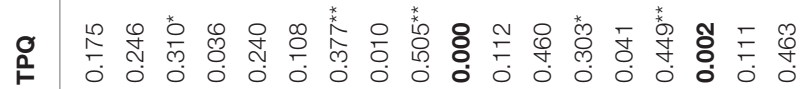

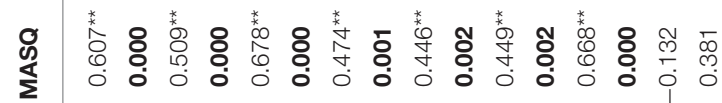

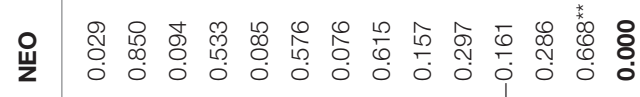

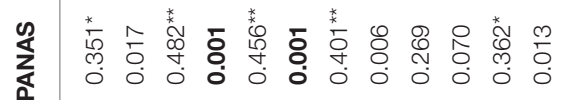

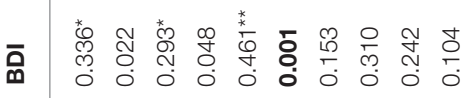

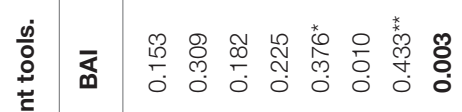

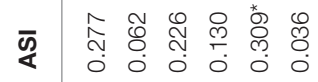

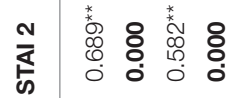

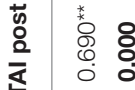

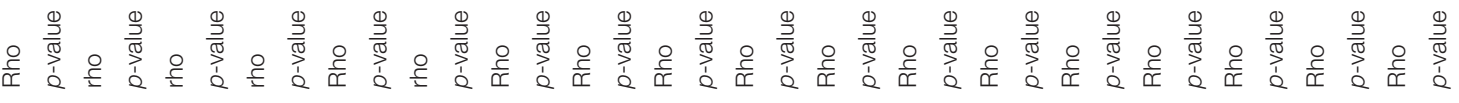

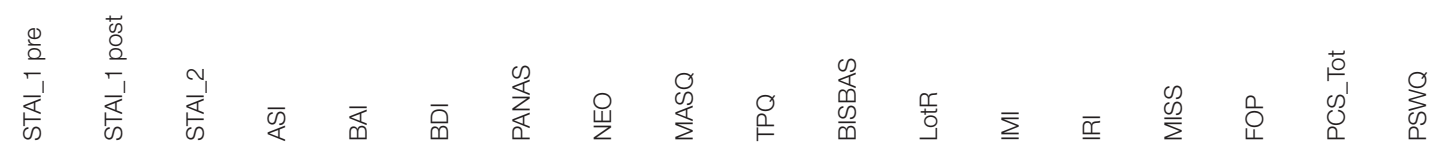


level of expectation related to pain changes (e.g., reductions and increases). Future large scale studies deserve to be performed in pain patient and healthy populations to better understand the connection among psychological factors, expectancies, placebo and nocebo effects.

The inclusion of an extensive battery of questionnaires related to personality factors allowed us to reveal that expectations may predict placebo and nocebo effects independently of personality factors making it a helpful tool for health care providers.

Several studies have emphasized the need for exploring the impact of personality factors as at least one of the possible ways to interpret and understand the large variability in placebo analgesic and nocebo hyperalgesic responses. To our knowledge, this is the first study that explores how distinct psychological factors can predict placebo hypolagesic responses and nocebo hyperalgesic responses, and the potential influence of personality factors in shaping positive and negative expectancies. Collectively, the complexity and variability in placebo- and nocebo-induced pain responses highlight a need to better understand the multidimensionality of pain and its modulation related to individual expectations and psychological factors. This

\section{REFERENCES}

Aslaksen, P. M., and Lyby, P. S. (2015). Fear of pain potentiates nocebo hyperalgesia. J. Pain Res. 8, 703-710. doi: 10.2147/JPR.S91923

Beck, A. T., Epstein, N., Brown, G., and Steer, R. A. (1988). An inventory for measuring clinical anxiety: psychometric properties. J. Consult. Clin. Psychol. 56, 893-897. doi: 10.1037/0022-006x.56.6.893

Beck, A. T., Ward, C. H., Mendelson, M., Mock, J., and Erbaugh, J. (1961). An inventory for measuring depression. Arch. Gen. Psychiatry 4, 561-571. doi: 10.1001/archpsyc.1961.01710120031004

Carver, C. S., and White, T. L. (1994). Behavioral inhibition, behavioral activation, and affective responses to impending reward and punishment: the BIS/BAS scales. J. Pers. Soc. Psychol. 67, 319-333. doi: 10.1037/0022-3514.67.2.319

Cloninger, C. R., Przybeck, T. R., and Svrakic, D. M. (1991). The tridimensional personality questionnaire: U.S. normative data. Psychol. Rep. 69, 1047-1057. doi: 10.2466/pr0.1991.69.3.1047

Colagiuri, B., Schenk, L. A., Kessler, M. D., Dorsey, S. G., and Colloca, L. (2015). The placebo effect: from concepts to genes. Neuroscience 307, 171-190. doi: 10.1016/j.neuroscience.2015.08.017

Colloca, L., and Benedetti, F. (2006). How prior experience shapes placebo analgesia. Pain 124, 126-133. doi: 10.1016/j.pain.2006.04.005

Colloca, L., and Benedetti, F. (2009). Placebo analgesia induced by social observational learning. Pain 144, 28-34. doi: 10.1016/j.pain.2009.01.033

Colloca, L., and Grillon, C. (2014). Understanding placebo and nocebo responses for pain management. Curr. Pain Headache Rep. 18:419. doi: 10.1007/s11916-014-0419-2

Colloca, L., Petrovic, P., Wager, T. D., Ingvar, M., and Benedetti, F. (2010). How the number of learning trials affects placebo and nocebo responses. Pain 151, 430-439. doi: 10.1016/j.pain.2010.08.007

Colloca, L., Sigaudo, M., and Benedetti, F. (2008). The role of learning in nocebo and placebo effects. Pain 136, 211-218. doi: 10.1016/j.pain.2008.02.006

Corsi, N., Emadi Andani, M., Tinazzi, M., and Fiorio, M. (2016). Changes in perception of treatment efficacy are associated to the magnitude of the nocebo effect and to personality traits. Sci. Rep. 6:30671. doi: 10.1038/srep30671

Costa, P. T., and McCrae, R. R. (1985). The NEO Personality Inventory Manual. Odessa, FL: Psychological Assessment Resources.

Costa, P. T., and McCrae, R. R. (1992). NEO PI-R Professional Manual. Odessa, FL: Psychological Assessment Resources.

Crawford, J. R., and Henry, J. D. (2004). The positive and negative affect schedule (PANAS): construct validity, measurement properties and normative approach provides advantages in interpreting how pain is felt and experienced.

\section{AUTHOR CONTRIBUTIONS}

LC and NC conceived the study design. NC performed the experiments. NC and LC analyzed the data and drafted the manuscript. LC revised and finalized the manuscript.

\section{FUNDING}

This research was partially supported by the University of Maryland, Baltimore (LC), the National Institute of Dental and Craniofacial Research (1R01DE025946-0; LC) and the Cooperint Internalization Program from University of Verona, Italy (NC).

\section{ACKNOWLEDGMENTS}

The authors thank Taylor Ludman, Andrea Howe, Maxie Blasini and Cynthia Renn for help with the set-up, screening of study participants or comments on the manuscript. data in a large non-clinical sample. Br. J. Clin. Psychol. 43, 245-265. doi: 10.1348/0144665031752934

Davis, M. H. (1980). A multidimensional approach to individual differences in empathy. JSAS Catalog Select. Doc. Psychol. 10:85.

De Pascalis, V., Chiaradia, C., and Carotenuto, E. (2002). The contribution of suggestibility and expectation to placebo analgesia phenomenon in an experimental setting. Pain 96, 393-402. doi: 10.1016/S0304-3959(01)00485-7

Flaten, M. A., Aslaksen, P. M., Finset, A., Simonsen, T., and Johansen, O. (2006). Cognitive and emotional factors in placebo analgesia. J. Psychosom. Res. 61, 81-89. doi: 10.1016/j.jpsychores.2005.12.004

Flor, H., and Turk, D. C. (1988). Chronic back pain and rheumatoid arthritis: predicting pain and disability from cognitive variables. J. Behav. Med. 11, 251-265. doi: 10.1007/BF00844431

Geers, A. L., Helfer, S. G., Kosbab, K., Weiland, P. E., and Landry, S. J. (2005). Reconsidering the role of personality in placebo effects: dispositional optimism, situational expectations, and the placebo response. J. Psychosom. Res. 58, 121-127. doi: 10.1016/j.jpsychores.2004.08.011

Geers, A. L., Helfer, S. G., Weiland, P. E., and Kosbab, K. (2006). Expectations and placebo response: a laboratory investigation into the role of somatic focus. $J$. Behav. Med. 29, 171-178. doi: 10.1007/s10865-005-9040-5

Geers, A. L., Kosbab, K., Helfer, S. G., Weiland, P. E., and Wellman, J. A. (2007). Further evidence for individual differences in placebo responding: an interactionist perspective. J. Psychosom. Res. 62, 563-570. doi: 10.1016/j.jpsychores.2006.12.005

Geers, A. L., Wellman, J. A., Fowler, S. L., Helfer, S. G., and France, C. R. (2010). Dispositional optimism predicts placebo analgesia. J. Pain 11, 1165-1171. doi: 10.1016/j.jpain.2010.02.014

Gelfland, D. M., Gelfland, S., and Rardin, M. W. (1965). Some personality factors associated with placebo responsivity. Psychol. Rep. 17, 555-562. doi: $10.2466 /$ pro.1965.17.2.555

Goubert, L., Crombez, G., and Van Damme, S. (2004). The role of neuroticism, pain catastrophizing and pain-related fear in vigilance to pain: a structural equations approach. Pain 107, 234-241. doi: 10.1016/j.pain.2003.11.005

Haigh, E. A., Moore, M. T., Kashdan, T. B., and Fresco, D. M. (2011). Examination of the factor structure and concurrent validity of the Langer Mindfulness/Mindlessness Scale. Assessment 18, 11-26. doi: $10.1177 / 1073191110386342$

Hayes, A. F., and Preacher, K. J. (2010). Quantifying and testing indirect effects in simple mediation models when the constituent paths are nonlinear. Multivariate Behav. Res. 45, 627-660. doi: 10.1080/00273171.2010.498290 
Huber, A., Lui, F., and Porro, C. A. (2013). Hypnotic susceptibility modulates brain activity related to experimental placebo analgesia. Pain 154, 1509-1518. doi: 10.1016/j.pain.2013.03.031

Hunter, T., Siess, F., and Colloca, L. (2014). Socially induced placebo analgesia: a comparison of a pre-recorded versus live face-to-face observation. Eur. J. Pain 18, 914-922. doi: 10.1002/j.1532-2149.2013.00436.x

Johnston, N. E., Atlas, L. Y., and Wager, T. D. (2012). Opposing effects of expectancy and somatic focus on pain. PLoS ONE 7:e38854. doi: 10.1371/journal.pone.0038854

Kotov, R. I., Bellman, S. B., and Watson, D. B. (2004). Multidimensional Iowa Suggestibility Scale (MISS) (New York, NY: Stony Brook University).

Kudel, I., Edwards, R. R., and Moric, M. (2005). The role of neuroticism, pain catastrophizing and pain-related fear in vigilance to pain: a structural equations approach. A comment on Goubert et al. (2004). Pain 115, 214-216; author reply 16-17. doi: 10.1016/j.pain.2005.02.008

Kube, T., Rief, W., and Glombiewski, J. A. (2017). On the Maintenance of Expectations in Major Depression - Investigating a Neglected Phenomenon. Front. Psychol. 8:9. doi: 10.3389/fpsyg.2017.00009

Lui, F., Colloca, L., Duzzi, D., Anchisi, D., Benedetti, F., and Porro, C. A. (2010). Neural bases of conditioned placebo analgesia. Pain 151, 816-824. doi: $10.1016 /$ j.pain.2010.09.021

Lund, K., Petersen, G. L., Erlandsen, M., De Pascalis, V., Vase, L., Jensen, T. S., et al. (2015). The magnitude of placebo analgesia effects depends on how they are conceptualized. J. Psychosom. Res. 79, 663-668. doi: 10.1016/j.jpsychores.2015.05.002

Lyby, P. S., Aslaksen, P. M., and Flaten, M. A. (2010). Is fear of pain related to placebo analgesia? J. Psychosom. Res. 68, 369-377. doi: 10.1016/j.jpsychores.2009.10.009

Markland, D., and Hardy, L. (1997). On the factorial and construct validity of the Intrinsic Motivation Inventory: conceptual and operational concerns. Res. Q. Exerc. Sport 68, 20-32. doi: 10.1080/02701367.1997.106 08863

Mehta, S., Rice, D., Janzen, S., Pope, J. E., Harth, M., Shapiro, A. P., et al. (2016). Mood, disability, and quality of life among a subgroup of rheumatoid arthritis individuals with experiential avoidance and anxiety sensitivity. Pain Res. Manag. 2016:7241856. doi: 10.1155/2016/7241856

Meyer, T. J., Miller, M. L., Metzger, R. L., and Borkovec, T. D. (1990). Development and validation of the penn state worry questionnaire. Behav. Res. Ther. 28, 487-495. doi: 10.1016/0005-7967(90)90135-6

Morton, D. L., Watson, A., El-Deredy, W., and Jones, A. K. (2009). Reproducibility of placebo analgesia: effect of dispositional optimism. Pain 146, 194-198. doi: 10.1016/j.pain.2009.07.026

Nes, L. S., and Segerstrom, S. C. (2006). Dispositional optimism and coping: a meta-analytic review. Pers. Soc. Psychol. Rev. 10, 235-251. doi: $10.1207 /$ s15327957pspr1003_3

Ober, K., Benson, S., Vogelsang, M., Bylica, A., Günther, D., Witzke, O., et al. (2012). Plasma noradrenaline and state anxiety levels predict placebo response in learned immunosuppression. Clin. Pharmacol. Ther. 91, 220-226. doi: 10.1038/clpt.2011.214

Osman, A., Breitenstein, J. L., Barrios, F. X., Gutierrez, P. M., and Kopper, B. A. (2002). The Fear of Pain Questionnaire-III: further reliability and validity with nonclinical samples. J. Behav. Med. 25, 155-173. doi: 10.1023/A:1014884704974

Peciña, M., Azhar, H., Love, T. M., Lu, T., Fredrickson, B. L., Stohler, C. S., et al. (2013). Personality trait predictors of placebo analgesia and neurobiological correlates. Neuropsychopharmacology 38, 639-646. doi: 10.1038/npp.2012.227

Petrovic, P. (2008). Placebo analgesia and nocebo hyperalgesia-two sides of the same coin? Pain 136, 5-6. doi: 10.1016/j.pain.2008.03.004

Preacher, K. J., and Hayes, A. F. (2004). SPSS and SAS procedures for estimating indirect effects in simple mediation models. Behav. Res. Methods Instrum. Comput. 36, 717-731. doi: 10.3758/BF03206553
Reiss, S., Peterson, R. A., Gurskey, D. M., and McNally, R. J. (1986). Anxiety sensitivity, anxiety frequency, and the prediction of fearfulness. Behav. Res. Ther. 24, 1-8. doi: 10.1016/0005-7967(86)90143-9

Rütgen, M., Seidel, E. M., Riecanský, I., and Lamm, C. (2015a). Reduction of empathy for pain by placebo analgesia suggests functional equivalence of empathy and first-hand emotion experience. J. Neurosci. 35, 8938-8947. doi: 10.1523/JNEUROSCI.3936-14.2015

Rütgen, M., Seidel, E. M., Silani, G., Riecanský, I., Hummer, A., Windischberger, C., et al. (2015b). Placebo analgesia and its opioidergic regulation suggest that empathy for pain is grounded in self pain. Proc. Natl. Acad. Sci. U.S.A. 112, E5638-E5646. doi: 10.1073/pnas.1511269112

Scheier, M. F., Carver, C. S., and Bridges, M. W. (1994). Distinguishing optimism from neuroticism (and trait anxiety, self-mastery, and self-esteem): a reevaluation of the Life Orientation Test. J. Pers. Soc. Psychol. 67, 1063-1078. doi: 10.1037/0022-3514.67.6.1063

Schweinhardt, P., Seminowicz, D. A., Jaeger, E., Duncan, G. H., and Bushnell, M. C. (2009). The anatomy of the mesolimbic reward system: a link between personality and the placebo analgesic response. J. Neurosci. 29, 4882-4887. doi: 10.1523/JNEUROSCI.5634-08.2009

Severeijns, R., Vlaeyen, J. W., van den Hout, M. A., and Weber, W. E. (2001). Pain catastrophizing predicts pain intensity, disability, and psychological distress independent of the level of physical impairment. Clin. J. Pain 17, 165-172. doi: 10.1097/00002508-200106000-00009

Spielberger, C. D. (1983). Manual for the State-Trait Anxiety Inventory STAI (form Y). Palo Alto, CA: Consulting Psychologist Press Inc.

Staats, P. S., Staats, A., and Hekmat, H. (2001). The additive impact of anxiety and a placebo on pain. Pain Med. 2, 267-279. doi: 10.1046/j.1526-4637.2001.01046.x

Sullivan, M. J., Bishop, S. R., and Pivik, J. (1995). The pain catastrophizing scale: development and validation. Psychol. Assess. 7, 524-532. doi: 10.1037/1040-3590.7.4.524

Swider, K., and Babel, P. (2013). The effect of the sex of a model on nocebo hyperalgesia induced by social observational learning. Pain 154, 1312-1317. doi: 10.1016/j.pain.2013.04.001

Trost, Z., Strachan, E., Sullivan, M., Vervoort, T., Avery, A. R., and Afari, N. (2015). Heritability of pain catastrophizing and associations with experimental pain outcomes: a twin study. Pain 156, 514-520. doi: 10.1097/01.j.pain.0000460326.02891.fc

Vogtle, E., Barke, A., and Kroner-Herwig, B. (2013). Nocebo hyperalgesia induced by social observational learning. Pain 154, 1427-1433. doi: 10.1016/j.pain.2013.04.041

Zubieta, J. K., Yau, W. Y., Scott, D. J., and Stohler, C. S. (2006). Belief or Need? Accounting for individual variations in the neurochemistry of the placebo effect. Brain Behav. Immun. 20, 15-26. doi: 10.1016/j.bbi.2005.08.006

Zuckerman, M. (1994). Behavioral Expressions on Biosocial Bases of SensationSeeking. New York, NY: Cambridge University Press.

Conflict of Interest Statement: LC has received lecture honoraria (Georgetown University and Stanford University) and has acted as speaker or consultant for Grünenthal and Emmi Solution. NC has no conflicts of interest to be declared.

The reviewer JG and the handling Editor declared their shared affiliation, and the handling Editor states that the process nevertheless met the standards of a fair and objective review.

Copyright $\odot 2017$ Corsi and Colloca. This is an open-access article distributed under the terms of the Creative Commons Attribution License (CC BY). The use, distribution or reproduction in other forums is permitted, provided the original author(s) or licensor are credited and that the original publication in this journal is cited, in accordance with accepted academic practice. No use, distribution or reproduction is permitted which does not comply with these terms. 